\title{
INTERPRETING SINGUIAR DEFINITE DESCRIPTIONS IN DATABASE QUERIES
}

\author{
Genevieve Berry-Rogghe \\ Department of Computer and Information Science \\ Temple University \\ Philadelphia, PA 19122
}

USÁ

\begin{abstract}
The paper examines some of the characteristic features of natural language interaction with a database systen and its implications for the processing of singular definite descriptions. Some proposals are made for assessing the uniqueness claim of the singular definite article in the context of retrieval from a relational database. Other standard assumptions such as the extensional evaluation and referent evaluation exclusively in the database - rather than within the discourse model - are critically examined.
\end{abstract}

\section{INTRODUCTION}

A comprehengive treatment of the phenomenon of singular definite description in natural language processing constitutes a research program par excellence in the fleld of cognitive science. Not only does it involve the various cognttive disciplines but also the integration of the traditional levels of analygis, namely the syntactic, semantic and pragmatic levels as well as including a "performance" or processing level dealing with mechanisms for memory managment of referring expressions. Iinguistic theories initially attempted to account for the syntactic conditions of well-formedness governing the introduction of singular definite nounphrases in sentences by postulating co-referentiality with a previously occurring indefinte nounphrase having the same referential index. But it became clear that the postulated requirements of co-referentiality could only be adequately stated by means of an extended notion of "discourse referent" (see Karttunen 1968 b). Loglcians and philosophers of language since Russell have studied problems of reference and of the loglcal form of referring expressions occurring in various contexts (for a selection, see Linsky 1971). But it was pointed out in Donnellan 1966 that a strictly truthfunctional analysis of singular definite expressions may in certain situations evaluate to a referent which was not intended by the speaker. Accounting for the intentions of the speaker introduces the pragmatic point of view of referring as a speech act (see Searle 1966). How the speaker proceeds in describing an object $8 /$ he has in mind so that it may be cor rectly identified by the hearer and how the hearer perceives the intentions of the speaker to accordingly process descriptions has been the object of psychologically oriented research in the area of definite reference (e.g. Clark/Marshal1 1981 and Ortony-Anderson 1977). In Artiflcial Intelligence, research on deIf it te descriptions has mainly proposed computational models to resolve anaphoric reference by postulating certain inference wechantsms (e.B. Charniak 1972 and Rleger 1974) or mechand sms for detecting and managing dialogue focus (e.g. Grosz 1981 and SIdner 1979).

Considering practical applications in the computer processing of natural language it way be said that next to none of this large body of research has been incorporated. Most natural language front-ends to a standard database system support none or very primitive reference resolution and treat the singular definite article as an existential quantifier. This state of affairs has frequently been motivated by the objection that most of the aforementioned research is not jet able to meet the challenge of a comoutationally efficient solution. It is hoped that such objections, though justified, (see for example Berwick 1983) will diminish in the course of time. More telling are objections relating to the nature of the natural language interaction with a database system which is alleged to constitute a restricted discourse environment not displaying the more sophisticated features of natural dialogue. The present paper critically examines some characteristics of such interactions with respect to the processing of definite descriptions and investigates to what extent the research findings aluded to above ought to be incorporated. The paper is intended to give an overview of the pro- 
blem domain rather than to offer concrete solutions; in fact its aim is to point out areas where frultful research is still outstanding.

\section{REFERRING TO DATABASE OBJECTS}

In a relational database an individual entity is defined by a unique tuple of attributes. The uni queness of individuals is guaranteed in the database scheme by declaring which attributes constitute the "key" of the relation. The key attributes as it were constitute the "essential" properties of an individual whereas its non-key attributes constitute 1ts "accidental" properties. (The term "individual" is used in a broad sense to denote abstract as well as physical objects, events, acts, situations ....). Philosophically, such properties are sald to be "essential" which uniquely characterize an individual in time and space - and remain constant in all "possible worlds". For example a human being might be essentially characterized by his/her parents, date and time of birth. In everyday discourse, on the other hand, individuals are usually referred to by a particular description which enables the hearer to identify the entity and which is chosen on the basis of assumptions about shared knowledge between the discourse participants. (See Clark/Marshall 1981 for a more detailed account of these assumptions).

When interacting with a database querying system a user may well be aware of the fact that 8 /he does not share any previous experience with the system and that it $1 \mathrm{~s}$ hence appropriate to characterize objects by their defining properties in the real world, le a sample is defined by the factory and location and the date and time it was taken and not by a description such as "the foul smeliing samplen. In principle then, the prospective user could be instructed to "use only fully specified descriptions". This is an unrealistic expectation for two reasons. First, in concrete database designs the key attributes of a relation are not usually chosen to reflect the state of affairs in the real world but rather out of considerations of processing efficiency. Second, the natural inclinition to be concise when interacting in natural language compels the user to use elliptical descriptions, as in the dialogue below :

- Where all samples taken from the firm Miller in 1980 analysed ?

- Yes.
- Did the sample taken in October contain any cyanide ?

Few users would remember to specify "from the firm M1Iler" in the follow-up query. A system which wishes to offer a minimum of comfort in use should be able to process "elliptical" descriptions which can be made unique by supplementing information from the preceding context.

So far we have assumed that in principle all entities a user wishes to refer to can be characterised by a number of essential properties. In reality the user often does not know the values for the essential attributes and hence has to use another description to refe: to the entity ; for example, "the sample from Miller that contained $250 \mathrm{mg}$ of arsentc". Such descriptions could be distinguished from the former by the fact that they contain some non-key attributes which the user claims happen to evaluate to a unique entity in a particular state of the database.

From the point of view of establishing the uniqueness of singular definite descriptions, one might distinguish the following four types :

(1) Intrinsically functional descriptions

(ii) extrinoically functional descriptions

(1ii) incomplete extrinsically functional descriptions

(iv) non-functional or reference establishing descriptions

Descriptions in category (i) are functional by virtue of the linguistic expression used. These include superlatives such as the highest salary, exprestions denoting aggregate functions such as the average, the sum .... and expressions with nominal modifiers such as the colour red and the number 13. Descriptions in category (11) are runctional by virtue of the state of the world (reflected in the database scheme and the integrity constraints). The president of the United States in 1985 , the sample from the flrm Miller taken on 13.10 .80 and the salary of G.B. Jones are examples of this catagory. Descriptions in category (iii) are incompletely specified instances of category (ii) which can be made functional by searching contextual information or by requesting such information from the user. Descriptions in category (iv) are sometimes called "reference establishing" as the specified properties do not guarantee the uniqueness of the description but the speaker maintains the description denotes uniquely and expects the hearer to accept 
this assumption.

In order to process descriptions efficiently a natural language processor should be able to recognize the type of a description. Intrinsically functional descriptions may be evaluated immediateIy in the database. For extrinsically functional descriptions the database scheme and the integrity constraints could indicate whether a given description is fully soecified. In case the description contains some but not all key attributes and it does not contain any non-key attributes it would seem to belong to category (iii) and in case it contains any non-key attributes it would appear to belong to category (1v). For cases of incomplete functional descriptions complementation procedures should be invoked, whereas the non-functional descriptions must be evaluated and an error message should be sent in case the description does not denote uniquely. Obviously, the proposals are only very tentative and require more thorough research. However, the bring us to consider the question of checking the uniqueness claim implicit in the use of singular the.

A natural language query to a relational database is usually translated into a query language based on the relational calculus and the singular defintte article is represented by the existential quantifier. For example, the que ry

- Who is the president of the D.S. married to ?

might be presented in a query languare based on the domain relational calculus a.s

$\{c / \exists \times$ PRES (PERSON $=x$, COONTRY $=$ 'USA') $\wedge$

MARRIED-TO (PERSON $\vec{z} x$, PERSON $=c)\}$

For a system to ignore the uni queness claim of the singular defint te article is at best uncooperative and at worst may lead to semantically incorrect representations. Thus, the distinction between restrictive and non-restrictive modifiers is ignored. (of courge the representation of non-restrictive modifiers introduces the additional problems of how to process surplus information). The following major proposals for the logical form of the singular definite article have been made : contextual elimination, a description operator and a special guantifier, Russell's proposal, namely $Q($ the $x$ Px) $\leftrightarrow \exists x$ Px $\wedge(\forall$ J $P y \rightarrow y=$ $x) \wedge$ Qx contextualiy eliminates the description, i.e. a description has no reference out of context. Russell's inclu- sion of the uniquenees postulate as a truth condition for the proposition implies that the sentence is false when the uniqueness claim fails. If the is treated as a description operator creating a singular term, the description may receive a denotation out of context. When the description has no referent, the proposition contains an undefined argument and on some interpretations lacks truth value. The treatment of the as a quantifier which takes a pair of predicates to form a sentence was advocated In Moore 1981 because it allows indication of scope differences. In the context of query evaluation it would seem more user-cooperative to treat propositions containing descriptions which do not evaluate to a unique referent as lacking truth value; thus, the uni queness claim might be viewed as a "semantic presuppostion". Onder the assumption that it is desirable for reasons of communicative adequacy to represent the relational claim in queries, it should be investigated how query languages based on the relational calculus could be augmented by a special quantifier or operator, given that the contextual elimination approach would create an excessive processing overhead.

ON THE PRAGMATICS OF REFERRING EXPRESSIONS

In natural discourse situations a speaker choses a description which will enable the listener to identify the speaker has in mind. In some contexts, however, "identification" is not to be interpreted in terms of the hearer retrieving some memory entity having the properties of the description. For example, a hearer may have been told: "the president of Zaire will be visiting France next weekn. Storing this proposition does not require finding a referent for the description the president of Zaire - although $s /$ he may do so depending on the hearer's world knowledge. It would appear that when processing statements containing descriptions, the hearer has the choice of elther resolving the referent or storing the description. (This is not the case in all contexts. For example, the statement "the woman who broke her leg is recovering" assumes previous knowledge of the referent, whereas the description the president of $\mathrm{Za}-$ ire presumably only presuposes the pre. vious knowledge that Zaire is a country and that countries may be governed by a president. (This aspect of the pragmatics of definite descriptions would be an interesting research topic, which to our knowledge has not been investigated) 
However, the speaker does not always leave the interpretation strategy up to the hearer but sometimes requires the hearer to identify the specific individual s/he has in mind, whereas in other contexts it may be the speaker's intention not to denote a specific individual but rather that the description be applied to the emantic referent. This is roughly the distinction made by "Donnellan between the "referential" and the "attributiven uses of the singular definite article.

Do the above observations about descriptions in statements equally apply to questions and 19 the referential/attributive distinction relevant in the context of database querying ? To answer this question comprehensively involves in the last resort epistimological considerations.

The question W1II the nresident of Zaire be visiting France might be answered positively by someone who happened to have read this news in the paper. Truth - functionally, howerer, the question can only receive a positive answer in case the corresponding statement represents a true proposition, after the description has receired an extensional evaluation, ie colonel Mobutu. The extersional approach to semantic evaluation is the one adopted in database querying. This strategy would seem approprlate in most cases. Suppose, however, the user asks the query "Can the president of the U.S. veto the Senate?" This description is not intended referentially (ie about Ronald Reagan in 1985) but attributively. It might be objected that the deacription in this context rather denotes a generic concept and that conventional database systems are not set up to answer such queries. Nevertheless, when a description has been extensionally evaluated the description itself should be retained for the subsequent resolution of anaphoric expressions. For example, in the following dialogue :

- Did the president of the D.S. visit Prance in 1982 ?

- No.

- Did he visit Germany in 1979 ?

Substitution of "Ronald Reagan" in the second query would result in a wrong answer. (Different interpretation modes for anaphoric descriptions have been investigated more thoroughly in Grosz et. al. 1983).
DISCOURSE REFERENTS IN DATABASE QUERIES

In the preceding section it was seen that a truth-functional evaluation of queries presupposes an extensional evaIuation of descriptions in the database. In natural discourse situations the referent of a description may be another description which appeared previously in the discourse, rather than an entity in the real world. This previously mentioned entity may be called a "discourse referent" a term introduced by Barttunen to explain certain phenomena of referential indexing for definite descriptions. According to Karttunen 68,a a discourse referent is nan entity that once it has been established - can be referred to by a pronoun or revived by a definite description" and is not to be equated with either "the individual the speaker has in mind" nor with "the thing in the real worldn. For example, the assertion

(s1) Jones took a sample from the flro Miller

establishes a discourse entity which may be revived by "the sample that Jones took from the firm Miller". Discour. se referents in the strict sense are introduced in the discourse by means of an indefinite description. (The notion has been extended in Kartinen $68 \mathrm{~b}$ ). Adopting the logical formalism for describing discourse referents proposed in Webber 1978 this discourge referent could be described as:

$$
(x: \operatorname{SAMPLE}(x) \hat{\text { TOOK }} \text { (Jones, } x)
$$

A proposition mentioning the sentence where the indefinite description was introduced establishes the contextual uniqueness of the singular definite description.

How does the notion of ndiscourse referent" as explained above operate in questions? For example, does the query

(S2) Did Jones take a sample from the firm Miller?

establish a discourse referent? Obviously, the description "the sample Jones took from the firm Miller" can only be used subsequently if the answer to (S2) is positive. It might be posited hence that the reply

(s3) Yes

implies the statement "Jones took a sample from the firm Miller" and that hance the description of the discourse referent would contain the proposition EVOKE $(x, S 3)$. 
A system which behaves like a natural discourse partner should store the description of the discourse referent (together with a pointer to its referential index which was retrieved on answering S2). If the user should in the subsequent discourse - no matter how many sentences intervene - refer to "the sample Jones took from Miller" the system should not reject this description as being incompletely specified.

If the answer to ( 52 ) had been negative, no discourse referent would have been established. Can one infer from this example the general rule that negatively answered questions cannot establish a discourse referent? Consider the following interaction :

(S4) Did the graduate admissions comittee hold a meeting today?

(S5) No.

(S6) Was the meeting postponed?

Can the expression "the meeting" in (S6) be construed as an abbreviation for "the meeting of the graduate admissions committee which was not held yesterday" or 1. it simply an abbreviation for $n$ the meeting of the graduate admissions committeen ? The difference between (S2) and (S4) can perhaps be accounted for by the difference between the non- opecific use of the indefint te article in (S2) and the specific use in (S4). In the specific use the relative clause derived from the predicate in the original question, ie that was held yesterday, should be understood in a non-restrictive sense and 18 hence not an essential part in the description of the discourse referent. Further research into the behaviour of indefinites in negative and interrogative contexts is is again called for.

\section{REFERENCES}

Berwlck R. (1983) Computational Aspects of Discourse. In : Computational Models or Discourse, Brady $M$. and Beiwick R. (eds), the MIT Press, 1983, 97-106.

Charniak I. (1972) Towards a model of children's story comprehengion, MI'T AI LAB TR-266, Cambridge MA, 1972.
Clark H. and Marshall C.M. (1981) Definite reference and mutual knowledge. In : Elements of Discourse Understanding, Joshi et al. (eds.), cambridge University Press, $1981,10-63$.

Donnellan K.S. (1966) Reference and definite descriptions. In : Philosophical Review, vol. 75, 1966,281 - 304 .

Grosz B., Joshi A.K. and Weinstein S. (1983) Providing a unified account of definite noun phrases in discourse, Proceedings of the $21 \mathrm{st}$ ACL, MIT, June $1983,44-50$.

Karttunen I. (1968b) What makes definite noun phrases definite? Technical report $\mathrm{P}-3871$, The Rand Corporation, June 1968.

Iinsky I. (ed.) (1971) Reference and Modal1ty, Oxford University Press.

Moore R.C. (1981) Problems in logical form, Proceedings of the 19th ACI, Stanford University, June 1981, 117-125.

Ortony A., Anderson R.C. (1977) Definite descriptions and semantic memory, Cognitive Science $1977,1,74-83$.

Rieger C.J. (1974) Conceptual memory:a theory and computer program for processing the meaning content of natural language utterances, stanford Artiflcial Inteligence Lab Memo AIM - 233, Stanford CA.

Searle J.R. (1969) Speech Acts, an Essay in the Philosphy of Language - New York; Cambridge University Press.

Sidner C. (1979) Towards a computational theory of definite anaphora comprehension in english discourse. Technical report 537. MIT Artificial Intelligence Laboratory, Cambridge MA

Webber B.I. (1978) A formal Approach to Discourse Anaphora. Technical report 3761, Bolt, Beranek and Newman, Cambridge MA. 